\title{
Research of lightning transient potential on the jacket foundation offshore wind turbines
}

\author{
Shiqi $\mathrm{Tao}^{1}$, Ximei $\mathrm{Yao}^{2}$, Bojing $\mathrm{Liu}^{3}$, Xiaoqing Zhang ${ }^{1}$ and Yaowu Wang ${ }^{l}$ \\ ${ }^{1}$ Beijing Jiaotong University, Department of Electrical Engineering, Beijing, the People's Republic of China \\ ${ }^{2}$ China Association for Standardization, Beijing, the People's Republic of China \\ ${ }^{3}$ China Electric Power Media Group, Beijing, the People's Republic of China
}

\begin{abstract}
Offshore wind turbines are often struck by lightning due to their tall structures and the harsh marine environment. The high transient potential from lightning strike can cause serious damage for the devices of offshore turbines. For analysing the effect of transient potential, a complete transient circuit model is established and an efficient algorithm is also presented to evaluate the circuit parameters of blade, tower, and jacket foundation. On the basis of the circuit model, the transient potential at the different locations of the offshore wind turbine can be carried out during direct lightning strike by PSCAD. Finally, the circuit model is used by a numerical example of an actual Chinese-built offshore wind turbine.
\end{abstract}

\section{Introduction}

Offshore wind turbines are especially susceptible to lightning strike because of their height and the marine environment. According to field observations [1], offshore wind turbines experience a significant number of lightning strikes during their lifetime. The lightning current will cause serious losses to the offshore wind turbine involves considerable costs of repair-materials, labour, and downtime. In recent years, the serious lightning accidents often appear in Hainan East Wind Farm in China, the damage rate of blades amounts to $5.56 \%$ [2]. As far as the lightning transient analysis of wind turbines is concerned, the existing works are only confined to the onshore wind turbines and the offshore wind turbines have not been undertaken [3-5]. Therefore, the research of lightning transient on the offshore wind turbines is very important to the lightning protection. In this paper, the complete transient circuit model is built for the jacket foundation offshore wind turbine and an efficient algorithm is also presented to evaluate the equivalent circuit parameters of the blade, tower and jacket foundation. The transient potential waveforms can be obtained on an actual Chinese-built offshore wind turbine.

\section{Transient circuit models}

\subsection{Lightning current}

As the current source injected into the tip of blade, the lighting current " $10 / 350 \mu \mathrm{s}$ " is used in transient analysis of offshore wind turbine [6], as show in Fig.1. The waveform of lightning current can be expressed by (1).

$$
i(t)=K I_{\mathrm{m}}\left(e^{-\alpha t}-e^{-\beta t}\right)
$$

where $I_{\mathrm{m}}$ is the peak value of lightning current and $K=1.025, \alpha=2.05 \times 10^{3}, \beta=5.64 \times 10^{5}$.

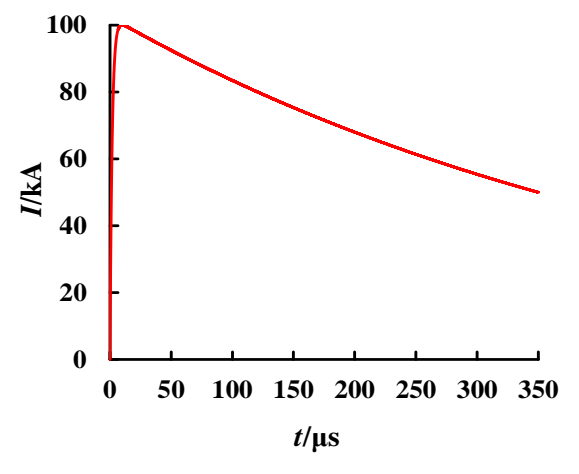

Fig. 1. Lightning current waveform.

The circuit model of the current source injected the jacket foundation offshore wind turbine is given in Fig. 2, where $Z_{0}(300 \Omega \sim 400 \Omega)$ is the surge impedance of the lightning channel [4].

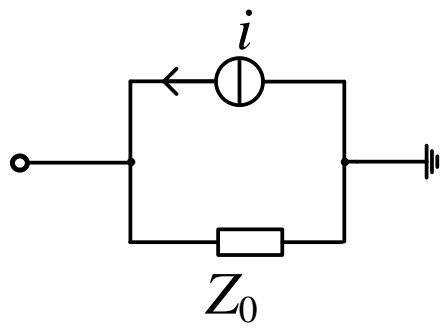

Fig. 2. Model of lightning current 


\subsection{Blade}

In general, the down conductor may be installed inside the blade for conducting lightning current. For the purpose of performing transient analysis, the down conductor needs to be divided into a number of segments to take account of the propagation wave phenomenon of lightning current, as shown in Fig.3(a) [7]. Each segment can be represented as a $\pi$-circuit consisting of resistance, capacitance and inductance, as shown in Fig.3(b).

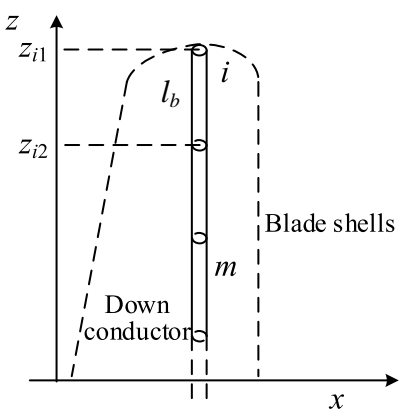

(a)

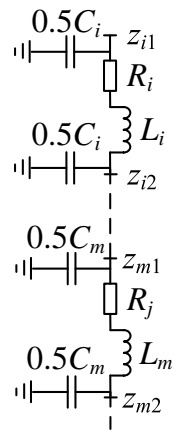

(b)
Fig. 3. Model of blade. (a)Schematic diagram of blade and down conductor, (b)Circuit model.

For arbitrary segment $b(b=1,2 \cdots m)$, its resistance is estimated as [8]

$$
R=\frac{\sqrt{\mu f} \Delta l_{b}}{2 r_{b} \sqrt{\pi \sigma}}
$$

where $r_{b}$ is the radius of the down conductor, $\mu$ is the material permeability, $\sigma$ is the material resistivity, $\Delta l_{b}$ is the length of each segment, $f$ may be roughly evaluated by the waveform parameters of the injected lightning current.

The capacitance of segment $b$ can be calculated by the average potential method [9]. Through complicated integral operation the capacitance of the segment $b$ is given by

$$
C_{i}=\frac{2 \pi \varepsilon_{0} \Delta l_{b}}{A_{1}+A_{2}}
$$

where

$$
\left\{\begin{aligned}
A_{1}= & \sinh ^{-1}\left(\frac{\Delta l_{b}}{r_{b}}\right)-\frac{\sqrt{r_{b}^{2}+\Delta l_{b}^{2}}}{\Delta l_{b}}+\frac{r_{b}}{\Delta l_{b}} \\
A_{2}= & \frac{1}{\Delta l_{b}}\left\{z_{i 1} \sinh ^{-1}\left(\frac{-2 z_{i 1}}{r_{b}}\right)+z_{i 2} \sinh ^{-1}\left(\frac{-2 z_{i 2}}{r_{b}}\right)-\right. \\
& \left(z_{i 1}+z_{i 2}\right) \sinh ^{-1}\left[\frac{-\left(z_{i 1}+z_{i 2}\right)}{r_{\mathrm{b}}}\right]+\frac{1}{2} \sqrt{r_{b}^{2}+4 z_{i 1}^{2}}+ \\
& \left.\frac{1}{2} \sqrt{r_{b}^{2}+4 z_{i 2}^{2}}-\sqrt{r_{b}^{2}+\left(z_{i 1}+z_{i 2}\right)^{2}}\right\}
\end{aligned}\right.
$$

The inductance $L i$ of segment $b$ is calculated by the Neumann's integral formula[10]:

$$
\begin{aligned}
L_{i}= & \frac{\mu_{0}}{4 \pi \Delta l_{b}}\left\{\Delta l_{b} \sinh ^{-1}\left(\frac{l_{b}}{r_{b}}\right)-\sqrt{\Delta l_{b}^{2}+r_{b}^{2}}+z_{i 1} .\right. \\
& \sinh ^{-1}\left(\frac{-2 z_{i 1}}{r_{b}}\right)+z_{i 2} \sinh ^{-1}\left(\frac{-2 z_{i 2}}{r_{b}}\right)-\left(z_{i 1}+z_{i 2}\right) . \\
& \sinh ^{-1}\left[\frac{-\left(z_{i 1}+z_{i 2}\right)}{r_{b}}\right]+\frac{1}{2} \sqrt{r_{b}^{2}+4 z_{i 1}^{2}}+ \\
& \left.\frac{1}{2} \sqrt{r_{b}^{2}+4 z_{i 2}^{2}}+r_{\mathrm{b}}-\sqrt{r_{b}^{2}+\left(z_{i 1}+z_{i 2}\right)^{2}}\right\}
\end{aligned}
$$

\subsection{Tower}

The actual tower of offshore turbine is a tubular circular truncated cone. In enginerring calculation, the tower body may be simplified as a cylindrical shell, as shown in Fig.4(a). In consideration of propagation wave phenomenon of lightning current traveling on the tower, the continuous cylindrical shell is approximately dissected into a number of segments [11] and each segment is represented by a $\pi$-circuit, as shown in Fig.4(b).

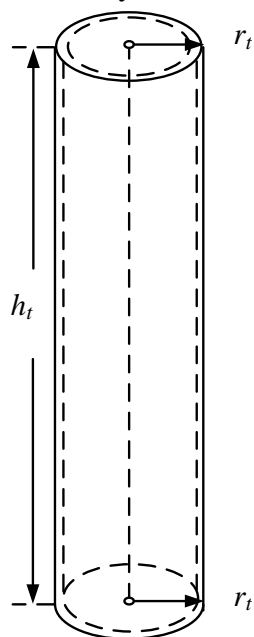

(a)

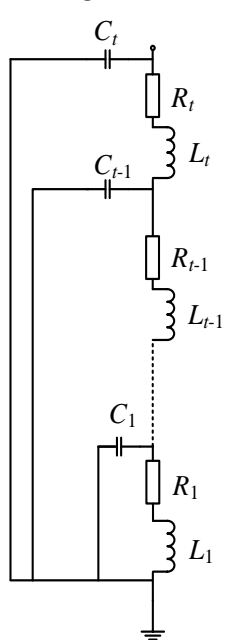

(b)
Fig. 4. Model of tower. (a)Schematic diagram of cylindrical shell tower, (b)Circuit model.

For an arbitrary segment $m(m=1,2 \cdots n)$, the resistance $R_{t}$ of segment $m$ is estimated by (5).

$$
R_{t}=\left\{1+\left(\sqrt{\frac{q}{2}}-1\right)\left[1-\frac{\omega}{r_{t}}-\frac{8}{4 \sqrt{2 q}-5}\left(\frac{\omega}{r_{t}}\right)^{2}\right]\right\} R_{0}
$$

where $q=2 \pi f \mu \sigma \omega^{2}, \mu$ is the material permeability, $\omega$ is the thickness of tower, $r_{t}$ is the equivalent outer radius of tower and $R_{0}$ is the $\mathrm{DC}$ resistance.

The capacitance $C_{t}$ of segment $m$ is calculated as

$$
C_{t}=\frac{2 \pi \varepsilon_{0} h_{t}}{\ln \frac{h_{t}}{r_{t}}-D_{\mathrm{m}}}
$$

where $h_{t}\left(h_{t}=h_{t} / n\right)$ is the length of segment $m, h_{t}$ is the height of tower, $\varepsilon_{0}=8.85 \times 10^{-12} \mathrm{~F} / \mathrm{m}$. $D_{\mathrm{m}}$ can be evaluated by [9].

The inductance $L_{t}$ of segment $m$ is calculated as [12] 


$$
L_{t}=\frac{\mu_{0} h_{t}}{2 \pi}\left(\ln \frac{2 h_{t}}{r_{t}}-1-\mu_{\mathrm{r}} \ln c\right)
$$

where $\mu_{\mathrm{r}}$ is the relative permeability of tower material, $\mu_{0}=4 \pi \times 10^{-7} \mathrm{H} / \mathrm{m}, c=\left(r_{t}-\omega\right) / r_{t}$.

\subsection{Jacket foundation}

As one of the fixed foundations, jacket foundation can effectively reduce the damage from bad marine environment, as shown in Fig.5[13]. When the offshore wind turbine is struck by lightning, the lightning current usually flows through the blade, tower and jacket foundation. Jacket foundation is soaked in the seawater, and the resistivity of seawater is far less than that of soil [14]. The seawater has a beneficial influence on dissipating the lightning current, so offshore wind turbines usually utilize jacket foundations as the natural grounding bodies.

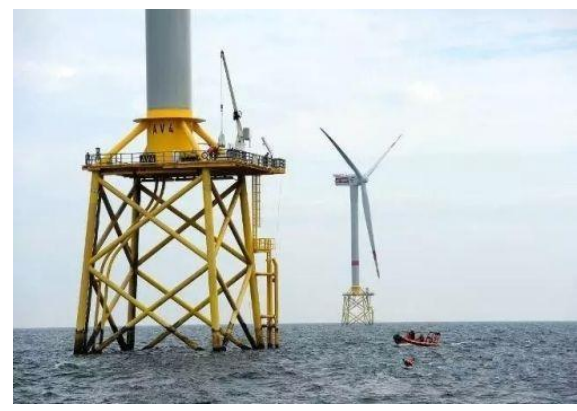

Fig. 5. Schematic diagram of offshore WT.

The whole jacket foundation can be equivalent to a grounding resistance. The grounding resistance of jacket foundation is affected by the seawater depth, clay layer thickness, seawater resistivity, clay resistivity, and gravel resistivity. From the requirement of analyzing the grounding resistance by CDEGS, the simulation model has been built by simplifying the hollow steel tubes into a series of solid steel cylinders, as shown in Fig.6.

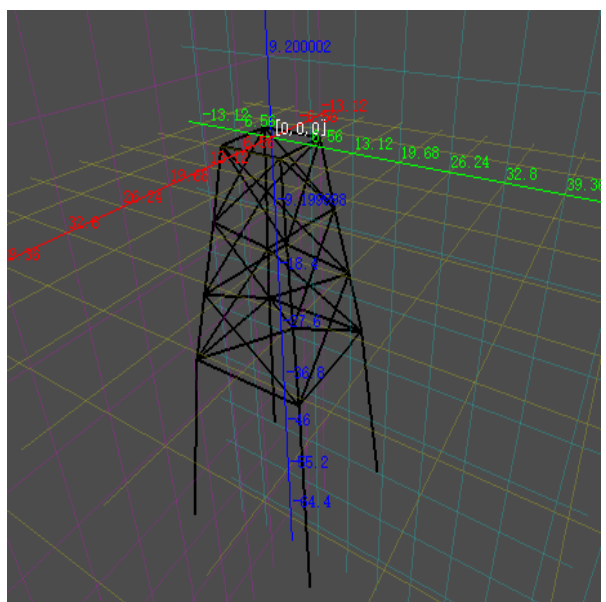

Fig. 6. Schematic diagram of offshore WT.

In this paper, when the seawater depth is $10 \mathrm{~m}$, clay thickness is $15 \mathrm{~m}$, seawater resistivity is $1 \Omega \cdot \mathrm{m}$, clay resistivity is $150 \Omega \cdot \mathrm{m}$ and gravel resistivity is $1000 \Omega \cdot \mathrm{m}$, the value of grounding resistance $R_{\mathrm{g}}$ is $0.227 \Omega$ by calculation.

After integrating the three separate equivalent circuit models described above, a complete circuit model can be built for the jacket foundation offshore wind turbine, as shown in Fig.7. Thus the waveforms of lightning transient potential can be obtained according to these transient circuit models.

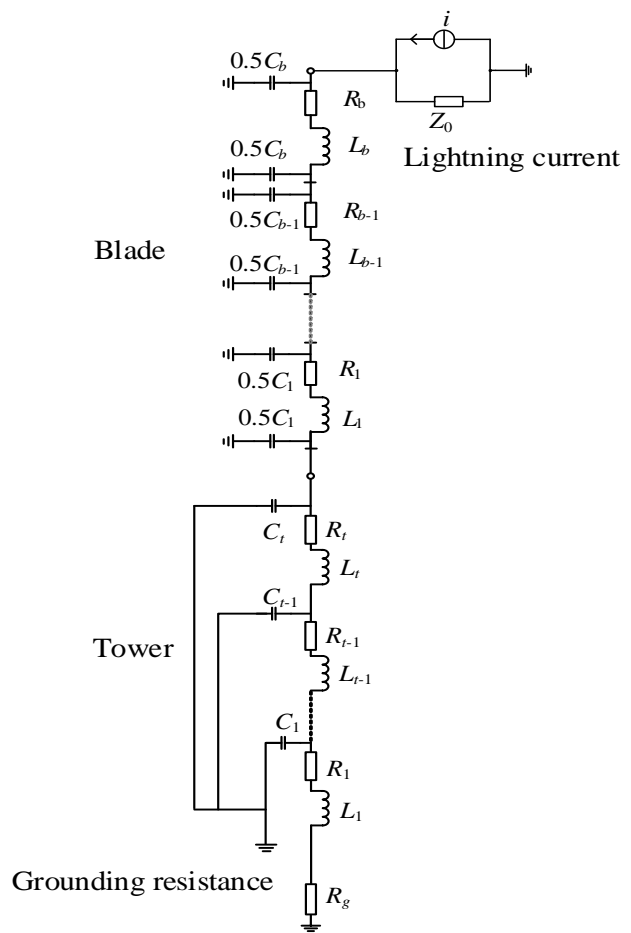

Fig. 7. Complete equivalent circuit model of wind turbine.

\section{Analysis of the transient potentials}

The dimensions of an actual Chinese-built offshore wind turbine are given Table 1. The transient potentials at different locations on the offshore wind turbine are obtained from the circuit model proposed above.

Table 1. Dimensions of an actual WT.

\begin{tabular}{ccc}
\hline & Parameters & Numerical value \\
\hline \multirow{2}{*}{ Blade } & Length $/ \mathrm{m}$ & 50 \\
& Down coductor radius $/ \mathrm{mm}$ & 4.7 \\
\hline \multirow{2}{*}{ Tower } & Height $/ \mathrm{m}$ & 70 \\
& Average thickness $/ \mathrm{mm}$ & 46 \\
& Top diameter $/ \mathrm{m}$ & 3.52 \\
& Bottom diameter $/ \mathrm{m}$ & 4.8 \\
\hline \multirow{2}{*}{ Jacket } & Seawater depth $/ \mathrm{m}$ & 10 \\
foundation & Clay layer thickness $/ \mathrm{m}$ & 15 \\
& Seawater resistivity $/ \Omega \cdot \mathrm{m}$ & 1 \\
& Clay resistivity $/ \Omega \cdot \mathrm{m}$ & 150 \\
& Gravel resistivity $/ \Omega \cdot \mathrm{m}$ & 1000 \\
\hline
\end{tabular}

The transient potential waveforms can be shown in Fig. 8 through the simulation software, PSCAD. In order to reflect the characteristics of transient waveforms, the 
two kinds of timelines will be used, respectively. Fig.8 is the long timeline and Fig.9 is the short one.

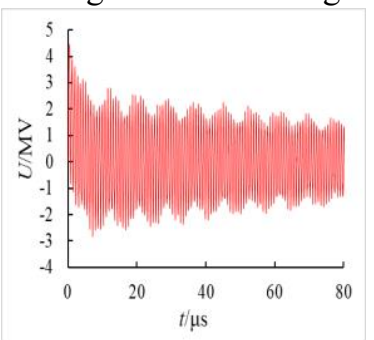

(a)

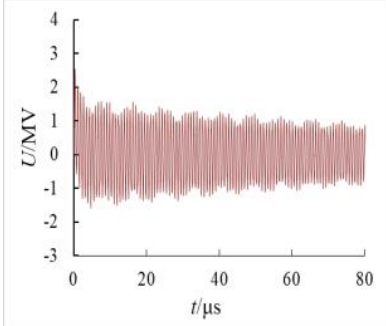

(c)

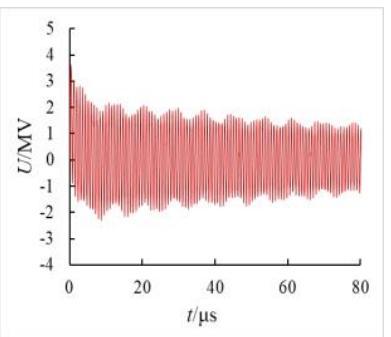

(b)

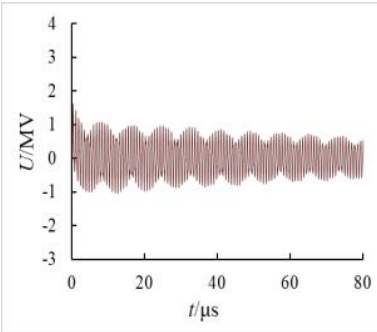

(d)

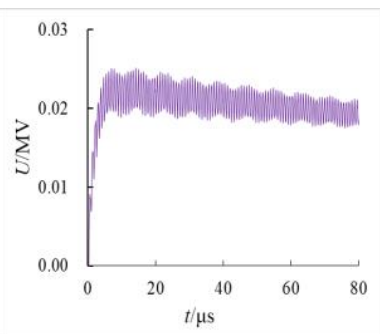

(e)

Fig. 8. Transient potential waveforms(long time scale).

(a) At the tip of blade, (b) At the middle of blade, (c) At the top of tower, (d) At the middle of tower, (e) At the bottom of tower.

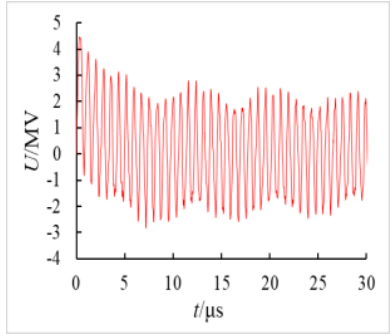

(a)

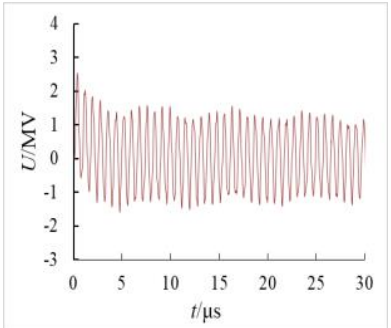

(c)

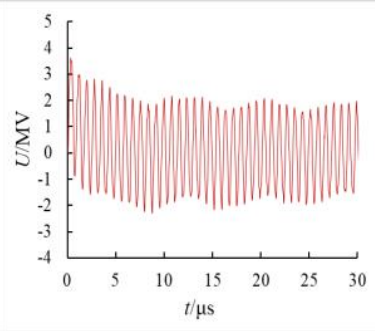

(b)

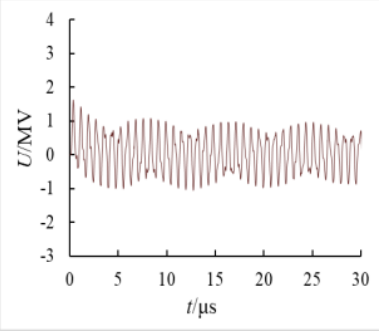

(d)

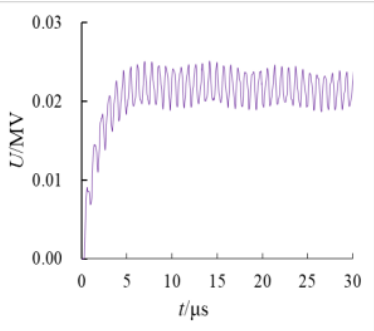

(e)

Fig. 9. Transient potential waveforms(short time scale). (a) At the tip of blade, (b) At the middle of blade, (c) At the top of tower, (d) At the middle of tower, (e) At the bottom of tower.

According to the simulation and calculation, the transient waveforms at some special positions(the tip of blade, middle of blade, top of tower, middle of tower, and bottom of tower) can be obtained. As shown in Fig.8 and Fig.9, the potential waveforms exhibit obvious oscillating behavior. This behavior directly results from the inductances and capacitances in the circuit models. It also indirectly embodies the reflection and refraction effects of the traveling waves. Finally, the potential waveforms will be convergent with increase in time.

As shown in Fig.8 (a) and (b), the waveforms of transient potential on the blade can be determined and it is essential for the lightning protection design. In terms of the potential difference between two specified points on the blade, the potential gradient can be estimated. Subsequently, the situation of creeping discharge on the blade can be further assessed. Finaly, a reasonable distance between the two receptors could be chosen to avoid the creeping discharge and thermal damage to the blade material. At the same time, the high transient potentials on the tower are liable to cause backflash to the facilities inside the tower during a lightning strike. Based on the waveforms of Fig.8 (c) and (d), the safe distance to avoid the backflash can be evaluated for installation of the facilities inside the tower. And as shown in Fig.8 (e), the potential at the bottom of tower will be raised for the existence of jacket foundation which may cause back flashover to the devices installed inside the tower. This high transient potential and sharp slope of waveform may destroy electric and electronic devices of the offshore wind turbines.

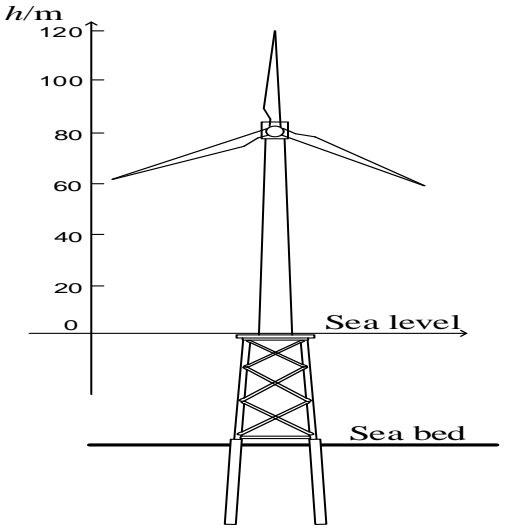

Fig. 10. The geometric position of the jacket foundation offshore wind turbine. 


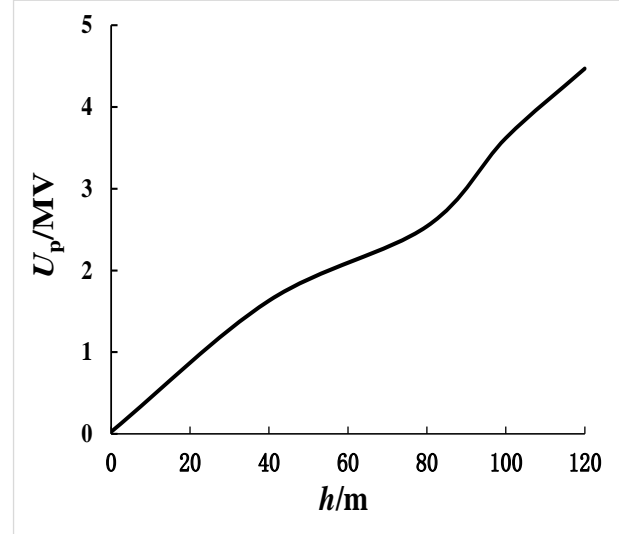

Fig. 11. Distribution of the transient potential peak values on the jacket foundation offshore wind turbine.

According to the Fig.10 and Fig.11, the distribution of the transient potential peak values on the jacket foundation offshore wind turbines can be obtained, and these peak values will decrease as the height decrease. The lightning protection design of offshore wind turbines can be considered according to these conclusions.

\section{Conclusions}

A complete circuit model has been proposed for analyzing the lightning transient potential of the jacket foundation offshore wind turbine. The model gives an overall description to the lightning current path from the blade tip to the jacket foundation. Using the model to perform transient calculation, the transient potential can be obtained at different locations on the offshore wind turbine. According to the numerical example that the lightning strikes to an actual offshore wind turbine, the peak value of transient potential at the blade tip will reach 4.47MV. Therefore these transient potentials rise on the offshore wind turbine is serious enough to do harm for the facilities and equipment of the offshore wind turbine, and the high transient potential needs to be considered to the lightning protection design of offshore wind turbines.

This work was partly supported by National Natural Science Foundation of China under Grant No. 51420105011.

\section{References}

1. Malcolm N, Aggarwal R. Analysis of transient overvoltage phenomena due to direct lightning strikes on wind turbine blade. IEEE Power \& Energy Society General Meeting. IEEE, 2014:1-5.

2. Li Qian, Zhong Dingzhu, Peng Xiangyang et al. Analysis of faults and lightning damage of wind power equipments of dongfang wind form second stage in Hainan province. Wind Power, 2002, (3): 22-27.

3. Xiang Xiao. Study on lightning overvoltage protection of wind turbines. Beijing Jiaotong University, 2016.

4. Wang X H, Zhang X Q. Modelling and Simulation of Lightning Transients in Wind Turbine Grounding Systems. Journal of System Simulation, 2010, 22(8):1805-1808.

5. X. Q. Zhang, A circuit approach to the calculation of lightning transients in cage-like multiconductor systems, International Journal of Electrical Engineering Education, Vol. 47 n. 2, April 2010, pp.213-222

6. IEC 61400-24, Wind turbine generator systems-Part 24: Lightning protection, 2010.

7. Rachidi $\mathrm{F}$, Rubinstein $\mathrm{M}$, Montanyà $\mathrm{J}$ et al. A review of current issues in lightning protection of new-generation wind-turbine blades. IEEE Transactions on Industrial Electronics, 2008, 55(6): 2489-2496.

8. R. B. Standler, Protection of electronic circuits from overvoltages (NY: John Wiley \& Sons, Inc., 1989, 68- 69)

9. Iosseli, A. S. Kothanof, M. G. Stlyrski, Calculation of capacitances (Moscow: Electric Power Press, 1987, 24-30)

10. C. R. Paul, Inductance-loop and partial (NJ: John Wiley \& Sons, Inc., 2010, 236-239)

11. Buccella C, Feliziani M. A hybrid model to compute the effects of a direct lightning stroke on three-dimensional structures. IEEE Transactions on Magnetics, 2003, 39(3): 1586- 1589.

12. P. L. Kalantrof, L. A. Ceitlin, Manual of inductance calculation (Moscow: Electric Power Press, 1992, 3-10)

13. Zhang Wei and Liu Jie, "Analysis of Platform of an Offshore Wind Turbine," International Journal of Electrical and Electronic Engineering \& Telecommunications, Vol. 6, No. 3, pp. 7-10, July 2017.

14. Gibson R E, Dowse B E W. The influence of geotechnical engineering on the evolution of offshore structures in the North Sea[J]. Canadian Geotechnical Journal, 2011, 18(2) 